Fecha de recepción: noviembre 2020 Fecha de aprobación: diciembre 2020 Fecha publicación: marzo 2021

\section{El arte visual en las tapas de las revistas Vogue y Harper's Bazaar del período de entreguerras}

Sara Peisajovich ${ }^{(1)}$

Resumen: Las preguntas acerca de la relación arte-moda se acumulan a lo largo de la historia. Infinitas son las aristas que se desprenden de este binomio. De hecho, muchos ejemplos que dan cuenta de la estrecha relación entre moda y arte son fácilmente constatables en algunas producciones culturales cotidianas, como las tapas de revista de moda. Analizando la discursividad y los diferentes sentidos de un conjunto de tapas del período de entreguerras, se retomarán las cuestiones más significativas de la relación entre las prácticas de las artes visuales, haciendo foco en las primeras vanguardias artísticas, y la moda de indumentaria para así comprender el modo por el cual ese fenómeno de la comunicación de moda, la tapa de revista, se configura en clave artística y evidencia el concreto vínculo entre un universo y el otro.

Palabras clave: moda - arte - comunicación - diseño - historia del arte - tapas de revistavanguardias artísticas

[Resúmenes en inglés y portugués en las páginas 165-166]

(1) Licenciada en Crítica de Artes, Universidad Nacional de las Artes. Docente UP. Docente UNA. Investigadora sobre temas de moda y arte.

\title{
Introducción
}

"Cuando la moda se vuelve un arte, una gazzette de la moda debe convertirse en una revista de arte. Y esa revista es La Gazzette du Bon Ton. La propia revista será una obra de arte” (Best, 2019).

Las preguntas acerca de la relación arte-moda se multiplican a lo largo de la historia. Infinitas son las aristas que se desprenden de este binomio y hacen foco en el fenómeno de lo artístico en la moda. Esto ocurre, en principio, porque muchos ejemplos que dan cuenta de la estrecha relación entre un universo y el otro son fácilmente constatables en algunas producciones culturales cotidianas, por ejemplo, en las tapas de revista de moda. 
Contemplar las tapas de Vogue y de Harper's Bazaar del período de entreguerras implica sumergirse en un nuevo modo de comunicar moda. Detenerse en los rasgos estilísticos, los motivos ilustrados, las técnicas empleadas y conociendo a los artistas que participaron de un conjunto de portadas de ese período histórico tan relevante para siglo XX permite analizar en detalle uno de los tantos entrecruzamientos entre las prácticas vestimentarias y diferentes lenguajes artísticos.

Sin ir más lejos y haciendo foco en uno de los ejes de este escrito, una vez más, la moda se apropia, en el buen sentido, de las prácticas y usos y costumbres de las artes visuales para retratar el espíritu de la época. Por medio de diferentes géneros pictóricos como el retrato o la naturaleza muerta y a través del uso del color, la composición, el dibujo a mano alzada o la acuarela, las tapas de revista se configuraron en clave artística y operaron como un discurso que, lejos de anclarse en lo frívolo o banal muchas veces asociado al sistema de la moda, ofrecieron un nuevo fenómeno producto del cruce entre ambos mundos.

La moda, al igual que el arte, es reflejo de su época y está atravesada por los avatares políticos, económicos, sociales y culturales del momento histórico en el cual se inscribe. En la indumentaria de cada período se perciben huellas de ello, en la silueta, la morfología, en los accesorios y en las tendencias que se fueron imponiendo, pero también en su modo de comunicar y en las diferentes prácticas vestimentarias que a su vez también mutaron y se reconfiguraron en relación al clima de cada época.

Si bien las tapas de revista son la puerta de entrada a ese mundo que se crea en las publicaciones de moda donde se dan a conocer los mencionados lineamientos generales del momento, las del período de entreguerras no se agotaron en esa única misión. También avanzaron en tematizar sobre la potencia artística de la moda. Moda entendida como ese sistema global consolidado a comienzos del siglo XX, como un producto ideológico de cada sociedad y cada cultura.

Esas revistas, además de tematizar sobre el vínculo entre moda y arte, pusieron en agenda esa potencialidad artística que se afirmó en la década de 1930 y creó novedosas posibilidades de producción en el campo de la comunicación de moda, al igual que en diseño. A su vez, esos gestos fueron retomados muchos años después por artistas y referentes de la fotografía de moda y la ilustración. Ese nuevo fenómeno que apeló al público bajo condiciones de producción netamente artísticas es ahora estudiado como un caso más dentro del binomio arte y moda.

En el debate acerca de si la moda es o no arte, estos discursos me servirán de ejemplo para avanzar hacia lo que considero más viable y enriquecedor con respecto a ese interrogante $y$ tiene que ver en primera instancia, con reconocer y analizar en particular cada caso de entrecruzamiento del arte y la moda. De ese modo, se podrá estudiar los fenómenos en profundidad para luego comprender en qué momentos aparecen esas prácticas vestimentarias que podrían ser analizadas desde una perspectiva artística.

Resulta más enriquecedor centrarse en cuándo es que se dan esos fenómenos de intersección entre una disciplina y la otra y sobre todo detenerse en el modo en el que se presentan. Indagar más profundamente en esas marcas artísticas permitirá, a partir de casos aislados y no tanto, establecer una posible generalidad que tenga como eje el vínculo entre determinadas prácticas del sistema de la moda y algunos lenguajes artísticos para avanzar 
y esbozar que no todo en la moda es arte pero sin duda muchas de sus acciones sí están intrínsecamente relacionadas.

En ese sentido, se retoma la línea de pensamiento del filósofo Nelson Goodman (1990) y su teoría que propone abordar al arte contemporáneo a partir de la pregunta de cuándo hay arte y no de qué es el arte, y se extrapola al campo de la moda. En sintonía con lo planteado por Goodman, se considera que será más fructífero comprender que el abismal sistema de la moda engendra infinitas posibilidades de producción y que si bien no todas comulgan con el arte, hay acontecimientos que sí lo hacen.

Entender esa estrecha relación es reconocer que la historia de la moda ofrece variados ejemplos donde el arte es parte fundamental de algunas prácticas vestimentarias. No sólo desde el punto de vista de inspiración de algún artista, o apropiándose de técnicas específicas, sino justamente sirviéndose del arte como medio, como soporte o lenguaje, imprimiéndole un sentido y una producción artística a diferentes discursos culturales, como en este caso, las tapas de revista.

Es a partir de allí que se indaga el gesto artístico de tales portadas y se avanza en el reconocimiento de determinados movimientos de vanguardia como el Surrealismo, el Cubismo o el Art decó. Entendiendo que los variados motivos presentes en las portadas articularon la temática propia del imaginario de la moda, como la vestimenta o el maquillaje, pero sin duda, se configuraron a partir del uso de técnicas de las artes visuales, grabando así el vínculo concreto entre un mundo y el otro.

\section{El marco histórico}

El período de entreguerras fue testigo de la creación de algunos de los movimientos artísticos más influyentes del siglo XX que fueron englobados en lo que se llamó las primeras vanguardias artísticas. Esas corrientes de las artes visuales y otras disciplinas como el teatro, la danza o el cine, se gestaron en Europa hacia finales del siglo XIX y se afianzaron a comienzos del siglo XX.

En un contexto bélico signado por las consecuencias de la Primera Guerra Mundial y la Revolución Soviética, así como también el surgimiento de regímenes totalitarios que provocaron el desenlace de la Segunda Guerra Mundial, sumado la gran depresión norteamericana de 1929 y el período de recesión económica que caracterizó ese momento histórico a lo largo y ancho del mapa, surgen estos movimientos de protesta que marcaron un antes y un después en la historia de la humanidad.

En el campo de las artes visuales esos movimientos artísticos forjaron estilos que se fueron consolidando a lo largo del tiempo para plantear una novedosa manera de hacer y sobre todo para ofrecer un legado estilístico que sería recuperado por otras disciplinas. Bajo el ala del modernismo, estos movimientos agitaron la bandera de lo rupturista y lo novedoso insertándose en lo que la historia del arte dio a llamar como las primeras vanguardias artísticas.

El término vanguardia, proveniente del francés, avant-garde, deriva del vocabulario militar. Se define como la primera posición, la más avanzada, lo adelantado de un ejército. Re- 
tomando esta acepción, los movimientos artísticos de principio de siglo XX simbolizaron no sólo la primera línea del cambio estético del momento, visionando un futuro artístico adelantado para la época, sino que habilitaron un legado estilístico que hasta el día de hoy es retomado por distintos lenguajes artísticos y otros sistemas afines.

Sumado a la definición etimológica del término vanguardia, se consolida otra arista dentro de ese término: la idea de promover el cambio, de habilitar la ruptura con lo anterior, de trascender el cánon. Así, promueven nuevos modos de hacer y configuran la idea de ruptura con la tradición. Es necesario comprender el concepto de vanguardia como capaz de revolucionar la existencia, la realidad tal como estaba dada para poder dimensionar el horizonte de posibilidades que prometía.

Uno de los modos de operar de estos movimientos fue a través del manifiesto. Los manifiestos artísticos fueron los escritos donde cada representante de los movimientos de vanguardia definía los lineamientos de su grupo. Estos estandartes expresaban los principios de cada uno, sus intereses. La figura del manifiesto me resulta fundamental en primer lugar, para comprender en profundidad tal período artístico. En segundo lugar, desde el punto de vista de la comunicación de moda y más precisamente de las tapas elegidas para este escrito. ¿Es que acaso esas portadas podrían operar como manifiestos del espíritu de la revista en analogía con el clima de época donde el arte conquistaba las prácticas vestimentarias?

En el campo de la moda, los mismos cambios sociales, políticos, económicos y culturales generaron modificaciones en el vestir. Las prendas se fueron adaptando tanto al paso del tiempo como al de una guerra tras otra. Los cuerpos fueron afinándose, lo voluptuoso ya dejó de estar de moda para dar lugar a las líneas más finas y delgadas y a cuerpos disimulados por su vestuario. Incluso, la vestimenta de guerra fue adoptada por la mujer en su casa y el diseño de indumentaria la tomó como necesidad en ese momento.

Quienes también se acoplaron al momento coyuntural y al aire artístico de la época, fueron las revistas de moda del período de entreguerras. El punto será analizar lo rupturistas que fueron y cómo trascendieron el cánon de lo esperable para sus portadas. Las fotografías, en reemplazo de los grabados en blanco y negro y a color de las primeras publicaciones de moda, quedaron en un segundo plano y determinadas prácticas de las artes visuales como el dibujo a mano alzada, la acuarela o el retrato, fueron dotando de un sentido creativo diferente al habitual a esos manifiestos de moda. De ese modo, otras formas de tematizar el universo femenino iban imprimiéndose en la historia de la comunicación de moda. Justamente el arte como herramienta de cambio, como instrumento para trastocar los límites impuestos, fue fundamental en las ediciones de Vogue y Harper's Bazaar de esos años. A partir de esas novedosas claves estilísticas, el cuerpo femenino fue retomado de infinitas formas para enmarcar una época y un momento histórico diferente. El binomio arte y moda quedó plasmado en el dispositivo tapa de revista que ofició de manifiesto dejando en claro que lo que se proponía era un despliegue creativo que acompañaba el clima de época. De hecho, un antecedente del vínculo entre arte y revista de moda fue $\mathrm{La}$ Gazette Du Bon Ton (1912-1925).

Creada por reconocidos diseñadores del momento, entre ellos Paul Poiret y Charles Frederick Worth, dos diseñadores paradigmáticos en lo relacionado a la consolidación del 
sistema de la moda a principios del siglo XX. La Gazette se enunciaba como un producto sin duda artístico diferenciándose así de las otras revistas del momento: "Los pintores colaboran con los modistos, la vestimenta femenina es un placer visual y, decididamente, no es inferior al resto de las artes" (Best, 2019, p. 320).

Sin duda fue Poiret el diseñador que más expresó ese clima artístico de la época, al igual que Elsa Schiaparelli y su dupla Salvador Dalí. Sólo para citar algunos referentes de ese mismo momento histórico donde las primeras vanguardias artísticas digitaban cada paso cultural de la sociedad, vale recordar los primeros pasos de Man Ray en la fotografía. El artista visual comenzó retratando las creaciones de Paul Poiret en clave surrealista y luego pasó a Harper's Bazaar de la mano de la mítica editora de moda Carmel Snow. Años más tarde la historia del arte lo inscribiría como uno de los artistas referentes del surrealismo. La década de 1930 fue un momento germinal para el vínculo entre moda y arte. Los casos surgidos en ese período fueron fundamentales para el devenir histórico del binomio arte-moda. Tanto las duplas de artistas y diseñadores, como la ilustración y fotografía de moda y sin dudas las tapas que van desde los años 20 hasta entrando en los 40, le allanaron el terreno a todo aquel que optara por servirse del arte para diseñar una colección, para darla a conocer, para hablar de ella o simplemente para experimentar su fusión y pronunciarse creativamente.

\section{Análisis de casos}

Ahora bien, para dar cuenta del modo por el cual algunas tapas de revista se hicieron eco del momento artístico y se presentaron como manifiestos evidenciando el poder visual de ese producto de la comunicación de moda, me detendré en algunos ejes presentes en cada una de ellas. Las variables atienden por un lado, a la cuestión más formal de la producción plástica como el uso del color, el estilo o la composición. Por otro lado, retomarán las características propias de algunos géneros consagrados en la historia del arte como el retrato o la naturaleza muerta. Se prestará también especial atención al gesto artístico de la firma del autor, sin descuidar la figuración de los cuerpos femeninos y al modo por el cual el universo de la moda es retratado en ese dispositivo.

Para ejemplificar las marcas artísticas de las tapas del corpus, comenzaré a describir cada una de ellas y eso permitirá evidenciar los variados rasgos relacionados con los movimientos de vanguardia y con las tendencias que invadían aquellos años. Lo primero que llama la atención es el uso del color como un recurso más que repetido. Las paletas brillantes y saturadas son las elegidas una y otra vez. Tal es el caso de la tapa de Vogue de febrero de 1936, donde un color puro inunda toda la extensión y sólo lo corta el logo de la revista en blanco (Figura 1).

Ese color azul ultramar (aunque si hubiese sido realizada treinta años después, a juzgar por sus pigmentos brillantes podríamos decir que es un azul klein), en plano completo, con el título en blanco y la indicación de la fecha y el precio sin más información, me recuerda los rasgos estilísticos de las pinturas del Suprematismo. 


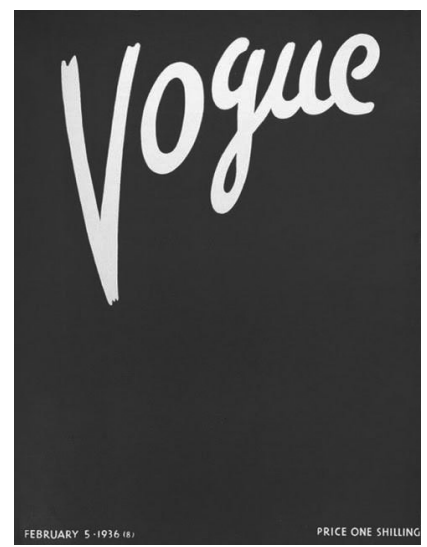
1

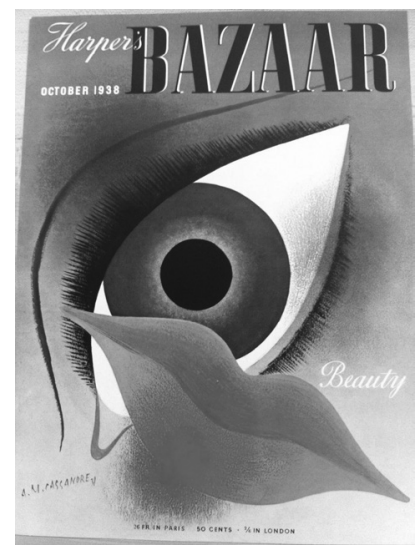

2
Figura 1. Tapa de Vogue 1936. Fuente: Revista Vogue, 1936. Figura 2. Tapa de Bazaar 1938. Fuente: Revista Harper's Bazaar, 1938.

El movimiento engendrado por Kazimir Malevich, artista ruso que trascendió los límites de la figuración y plasmó su percepción del mundo a través de la abstracción, propone volver al grado cero de la pintura, reducida a la parte más elemental del cuadro, las pinceladas, la huella del pintor e imprime en sus lienzos el color puro rojo, en la obra titulada: Cuadrado rojo de 1915 o el puro negro, en la obra titulada: Cuadrado negro, de 1923.

Volviendo al ejemplo, es una tapa vacía, sin contenido referencial a la moda o a la indumentaria. Se alude a la máxima simplificación de la representación de la realidad y sólo se percibe el estímulo abstracto de un color puro. Es que el estilo abstracto también fue reflejado por la moda y no podía quedar afuera de uno de los soportes más efectivos a la hora de marcar tendencia. Este guiño concreto a las artes visuales deja en claro también la intención de la revista de ser símbolo de la modernidad y de la voluntad de mostrar su sensibilidad artística.

Como explican Croci y Vitale (2011) en el texto: Los cuerpos dóciles, hablan en relación a las nuevas estéticas y siluetas de mujer comprometidas con la depuración y la abstracción asociadas al arte moderno. Se desarrolla en el texto cómo el arte moderno moldeó estéticamente a gran parte de la sociedad bajo los lineamientos de un cuerpo más longilíneo en sintonía con las características artísticas de ese momento.

Además de la abstracción y el uso del color, la técnica como la acuarela o el dibujo a mano alzada también formaron parte de la composición de las gráficas. En la portada de Harper's Bazaar de octubre de 1938 se aprecia el modo por el cual se configura el universo femenino a partir de la ilustración de un ojo y una boca bien maquillados. En este caso, la figura del cuerpo femenino, que como podemos observar no siempre es figurado de pies a cabeza, es retomado en forma de sinécdoque, entendiendo al ojo femenino bien maquillado en tonos azules en primer plano, sin más que un fondo de color anaranjado que contrasta con el maquillaje, como la parte del todo femenino (Figura 2). 


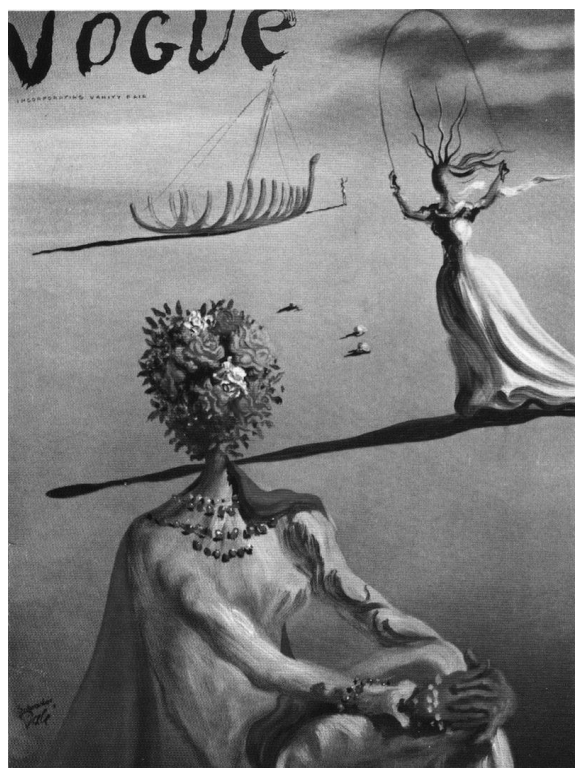

3

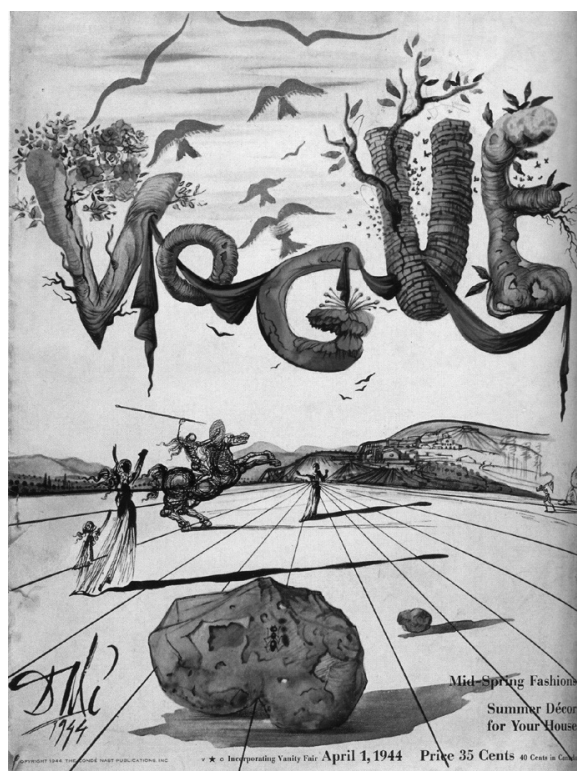

Figura 3. Tapa de Vogue 1938. Fuente: Revista Vogue, 1933. Figura 4. Tapa de Vogue 1944. Fuente: Revista Vogue, 1944.

El efecto que logra este diseño podría evocar los lineamientos surrealistas, aquellos que se guiaban por la irracionalidad, la ensoñación y la libertad creadora. El primer manifiesto surrealista de André Bretón lo define como "automatismo psíquico puro por cuyo medio se intenta expresar tanto verbalmente como por escrito o de cualquier otro modo el funcionamiento real del pensamiento" (Bretón, 2012, p. 44).

Es justamente ese atributo onírico propio del ojo dibujado en proporciones exageradas que se ve tapado por una boca de similar escala y que juntos crean una composición extraña o infrecuente que no dice mucho pero a la vez es suficiente para exacerbar ese concepto de belleza y tematizar sobre el universo de la cosmética. Allí se aborda lo artístico desde el punto de vista de la técnica como el dibujo a mano alzada pero también se evidencia una característica propia de uno de los movimientos de vanguardia del período de entreguerras, el Surrealismo.

A propósito de mencionar el aspecto surrealista en las portadas de Vogue y Harper's Bazaar, las portadas realizadas por el artista visual Salvador Dalí, tanto la de 1939 como la de 1944, no sólo se inscriben bajo los lineamientos de ese movimiento de vanguardia sino que también responden a una de las características que considero fundamental en el vínculo entre sendos universos: la firma del artista (Figuras 3 y 4 ). 
La firma de Dalí en ambas portadas no intenta informar quién las realizó, ya que sería una obviedad a juzgar por el estilo de la imagen, sino que encarna uno de los gestos artísticos por excelencia. La firma le concede a la tapa de Vogue de abril de 1944 el carácter de obra pictórica y lo diferencia de cualquier otra portada de moda. Esa tendencia de firmar el lienzo va a ser adoptada por todos los ilustradores que participen de las tapas de ese período en analogía con la obra de arte que cuelga de una pared.

Al observar la composición de la portada de 1944, lo que más llama la atención es el logo de la revista en un tamaño muy superior a los cuerpos que aparecen a lo lejos. Las diferentes escalas entre los objetos, entre esos cuerpos longilíneos y el logo recargado de detalles florales, cintas y plumas, articulan la pluralidad de motivos de indumentaria y, a su vez, son claros ejemplos del universo de Dalí y de las propiedades del surrealismo.

Continuando con el autor de la tapa anterior y la acción de elegir artistas consagrados del momento o Los Beau Brummell del pincel, como los llamó Condé Montrose Nast, Giorgio de Chirico, Joan Miró, Eduardo García Benito, entre otros, inmortalizaron sus diseños y despuntaron un recurso más de apropiación de las prácticas artísticas. Cabe destacar que esta modalidad continuó en décadas posteriores, cuando Joan Miró imprimió su sello para la revista a finales de la década del '70. Así también lo hizo Andy Warhol en la década de 1980.

Retomando el gesto de la firma del artista en las tapas, lo mismo ocurre en la Figura 5, donde en el margen inferior derecho aparece la firma del artista visual Giorgio de Chirico. Con su trazo retrató uno de los escenarios femeninos: el camarín, la habitación o el cambiador detalladamente ilustrado con la misma característica surrealista de situarlos en fondos irreales y a escalas no tradicionales. La firma, además de informar quién realizó la ilustración, predispone al lector a guardarla y hasta a enmarcarla, como pasa en la actualidad donde muchas de esas portadas son láminas listas para colgar de una pared y contemplarlas como obras de arte (Figura 5).

Si bien en esa tapa no se muestra ningún cuerpo de mujer, el universo femenino queda establecido sin lugar a dudas, habilitando así, otra forma de tematizar esa figura femenina a partir de la identidad de los movimientos artísticos de vanguardia. Esas mismas portadas surrealistas escapan al intento antiguo de hacer del arte un mero reflejo de la realidad.

Por el contrario, evocan al modernismo bajo la línea de enfatizar la construcción de autorreferencia en vez de ser el espejo de la sociedad. Edifican el momento por medio de la construcción de un universo onírico, irreal, artificial, compuesto de yuxtaposiciones, simultaneidad y montaje en sus objetos. La gráfica de moda necesitó de esa influencia artística para poder visibilizar lo que en su indumentaria también surgía de modo paralelo. A su vez, me resulta significativo atender a la cuestión de cómo el cuerpo será representado de mil y una posibilidades para dar a conocer las novedades de la indumentaria. En el caso de la tapa ilustrada por De Chirico, el primer plano del collar, los guantes y esa rosa blanca simulan la composición de una naturaleza muerta, pero con los típicos accesorios femeninos en reemplazo de la fruta, la copa o los otros objetos típicos de la pintura de bodegón.

Hasta aquí vimos cómo el universo femenino fue representado desde la perspectiva abstracta del color puro o bajo los lineamientos de uno de los movimientos más destacados de las primeras vanguardias artísticas. También lo vimos fracturado y delineado a mano 

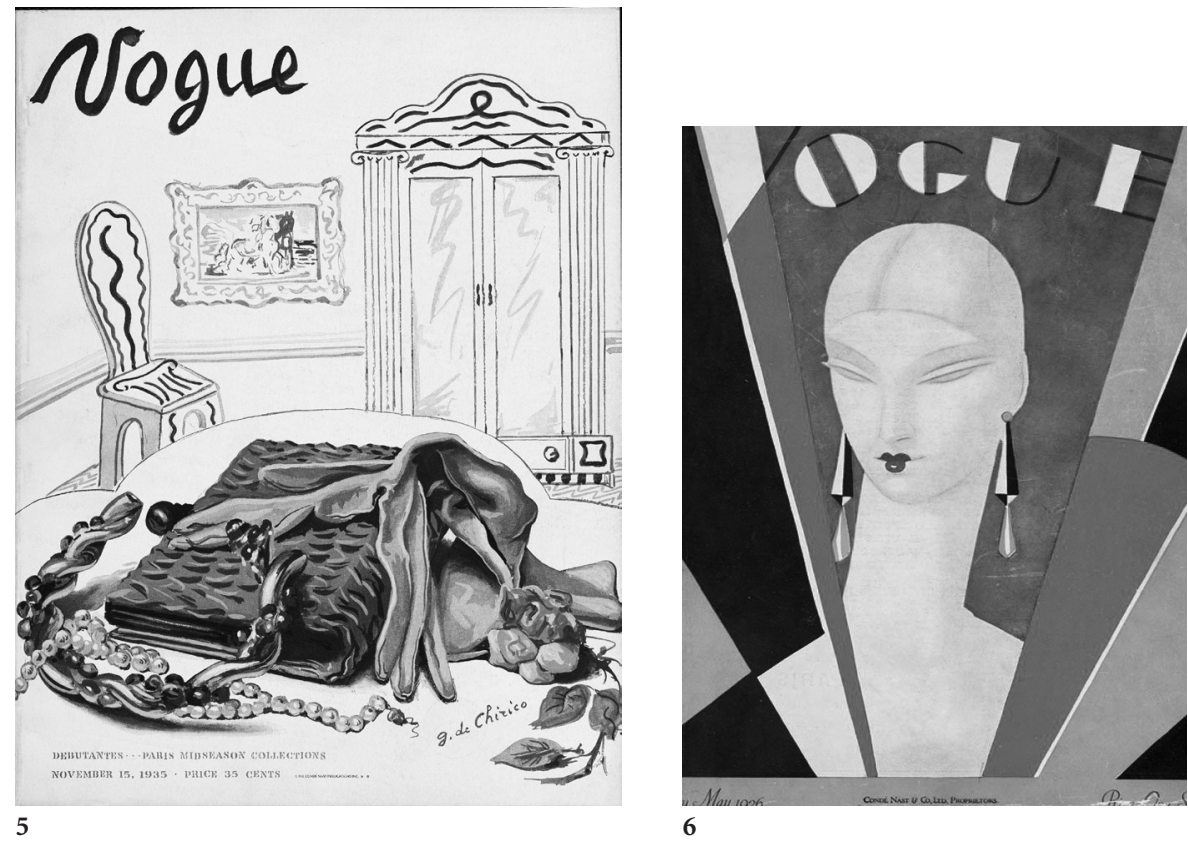

Figura 5. Tapa de Vogue 1935. Fuente: Revista Vogue, 1935. Figura 6. Tapa de Vogue 1926. Fuente: Revista Vogue, 1926.

alzada, haciendo hincapié en una de sus partes constitutivas o como motivos de una clásica naturaleza muerta. Sin duda las posibilidades artísticas del momento fueron el leitmotiv de ese deseo de promover lo que iba ocurriendo en la moda y el arte del momento. Es a partir de ahí que las tapas del período de entreguerras hacen uso de las prácticas de las artes visuales retomando esos fundamentos y procesándolos en clave vanguardista.

Ahora bien, continuando con la descripción de las portadas y observando la tapa de Eduardo de Benito para la edición de mayo de 1926 de la revista Vogue, se percibe un retrato típico del Art decó. Este movimiento caracterizado por una estética clásica, rectilínea y bien simétrica, no sólo fue propio de las artes visuales sino de la arquitectura y la ilustración de moda. Su mayor exponente fue Eduardo García Benito quien también retomaría otras influencias estilísticas como las del Cubismo de Pablo Picasso y las estilizadas figuras del pintor y escultor italiano Amadeo Modigliani (Figura 6).

Benito dibujó los retratos colocando en primer plano los cuellos largos de esas mujeres muchas veces dibujadas con rasgos orientales. Las formas geométricas y los ángulos cubistas también inundaron sus creaciones. Las paletas de colores incluyeron una amplia gama de pigmentos, algunos más opacos y otros bien brillantes, pero privilegiando la termina- 
ción sólida de pocos colores en cada pintura. Sus mujeres bien elegantes y refinadas fueron referente de infinitas portadas.

La fragmentación y descomposición de los planos y las formas geométricas componen el busto femenino de la Figura 5. Sus rasgos más significativos son la dislocación del cuerpo y los ángulos definidamente marcados. La edición de Vogue de mayo de 1926 responde a esos lineamientos del Cubismo y el Art Decó a la perfección. A propósito de este último, el logo de la revista no es un detalle menor. Se puede observar como el comienzo del logo está mimetizado con el trazo diagonal que enmarca y delimita el retrato femenino.

Así, todos los componentes del dispositivo tapa de revista destilaban arte. En ningún caso el logo Vogue o Harper's Bazaar quedaba separado del estilo reinante, sino que se trataba de una relación directamente proporcional entre éste y el estilo elegido para cada edición. Se compartían la misma paleta de color o similar tipografía para no desentonar con la creación y formar parte del todo final que sería la Cover Art para el recuerdo. Vale aclarar que el período de las Cover Art se ubica de 1920 a 1960 según explica Gerald Grow en su artículo Magazine Covers and Cover lines: an illustrated history. Fueron portadas protagonistas que evitaron la inclusión de títulos, subtítulos y texto adicional que interfiera con el diseño general como es usual en la actualidad del siglo XXI.

\section{Conclusión}

A lo largo de estas páginas se evidenció el modo por el cual las tapas seleccionadas pusieron de manifiesto su proceso de producción íntimamente relacionado con algunas prácticas de las artes visuales, gestionando el contacto con sus lectores a partir del lenguaje plástico. De hecho, la modalidad de simular el cuadro pictórico llegó hasta casi todos los niveles, como si se buscara no entorpecer la visión de la pieza artística con nada del exterior, o del interior, ya que la tapa oficia como puerta de entrada de ese contenido que el público ya conoce y que se despliega en el interior de cada publicación.

En definitiva, la recurrencia de las técnicas empleadas contribuyó a modificar las preferencias de las gráficas de moda. Le otorgó la libertad que estaba necesitando, eso que antes no existía, donde todo era fotografía o figurines. Las tapas elegidas se volvieron manifiestos. Emblemas de un clima de época que posibilitaron decodificar el universo de la moda a partir de las artes visuales. Porque los valores morales y estéticos de cada momento histórico pueden ser reconocidos fácilmente tanto en las vestimentas como en las obras de arte y son estas tapas de revista de Vogue y Harper's Bazaar del período de entreguerras las que a modo de agentes catalizadores dieron cuenta de ello y se enunciaron como manifiestos postulando para la eternidad el vínculo concreto entre arte y moda.

\section{Referencias bibliográficas}

Barthes, R. (2008). El sistema de la moda. Argentina: Paidós Comunicación. 
Beaton, C. (1990). El espejo de la moda. Barcelona: Parsifal Ediciones.

Benjamin, W. (1967). El arte en la era de su reproductibilidad técnica. Buenos Aires: Nueva Visión.

Bermejo, J. M. (1995). Kasimir Malevich. Barcelona: Ediciones Polígrafa.

Bragagnolo, M. (1991). ¿Qué es el arte del siglo XX? Madrid: Granada E y D.

Breton, A. (2012). Manifiestos del surrealismo ( $3^{\mathrm{a}}$ ed.). Buenos Aires: Argonauta.

Croci, P. y Vitale, A. (1992). Los cuerpos dóciles. Buenos Aires: la marca editora.

Grow, G. (2002). Magazine Covers and Cover Lines: An illustrated history, Journal of Magazine \& New Media Research 5, 1.

Goodman, N. (1990). Maneras de hacer mundos. Madrid: Visor distribuciones.

Lipovetsky, G. (2014). El imperio de lo efímero. Barcelona: Anagrama.

Morgan, R. (1998). El fin del mundo del arte y otros ensayos. Argentina: Eudeba.

Nelson Best, K. (2019). El estilo entre líneas. Buenos Aires: Ampersand.

Pinto, F. y Cancela, D. (2004). Moda para principiantes. Buenos Aires: Era Naciente.

Saulquin, S. (2014). Política de las apariencias: nueva significación del vestir en el contexto contemporáneo. Buenos Aires: Paidós.

Steel, V. (2018). Fashion theory: hacia una teoría cultural de la moda. Buenos Aires: Ampersand.

Tatavitto, S. (2009). Historias de las tapas de revista: Magazine Cover and Cover Line. An illustrated History y 100 years of Magazine covers, Revista Figuraciones, Disponible en: http://repositorio.una.edu.ar/handle/56777/530.

Abstract: Questions about the art-fashion relationship accumulate throughout history. Infinite are the edges that emerge from this binomial. In fact, many examples that show the close relationship between fashion and art are easily seen in some everyday cultural productions, such as the covers of a fashion magazine. Analyzing the discursiveness and the different meanings of a set of tapas from the interwar period, the most significant questions of the relationship between the practices of the visual arts will be retaken, focusing on the first artistic avant-gardes, and clothing fashion in order to understand the way in which this phenomenon of fashion communication, the magazine cover, is configured in an artistic key and shows the concrete link between one universe and the other.

Keywords: fashion - art - communication - design - art history - magazine covers - artistic vanguards.

Resumo: Perguntas sobre a relação arte-moda se acumulam ao longo da história. Infinitas são as arestas que emergem desse binômio. Na verdade, muitos exemplos que mostram a estreita relação entre moda e arte são facilmente vistos em algumas produções culturais do cotidiano, como as capas de uma revista de moda. Analisando a discursividade e os diferentes sentidos de um conjunto de tapas do período entre guerras, serão retomadas as questões mais significativas da relação entre as práticas das artes visuais, enfocando as 
primeiras vanguardas artísticas e a moda do vestuário para compreender a forma como este fenômeno de comunicação da moda, a capa da revista, se configura em tom artístico e mostra a ligação concreta entre um universo e outro.

Palavras chave: moda - arte - comunicação - design - história da arte - capas de revistas - vanguardas artísticas.

[Las traducciones de los abstracts fueron supervisadas por el autor de cada artículo] 